\section{Modeling of Intense Line Radiation from Laser-Produced Plasmas}

UCRL-JC-113605

PREPRINT

Yim T. Lee and M. Gee

Lawrence Livermore National Laboratory

Livermore, CA 94550

This paper was prepared for submittal to Conference Proceedings of International Workshop on Laser-Plasma Interaction

November 10-15, 1989

Monterey, California

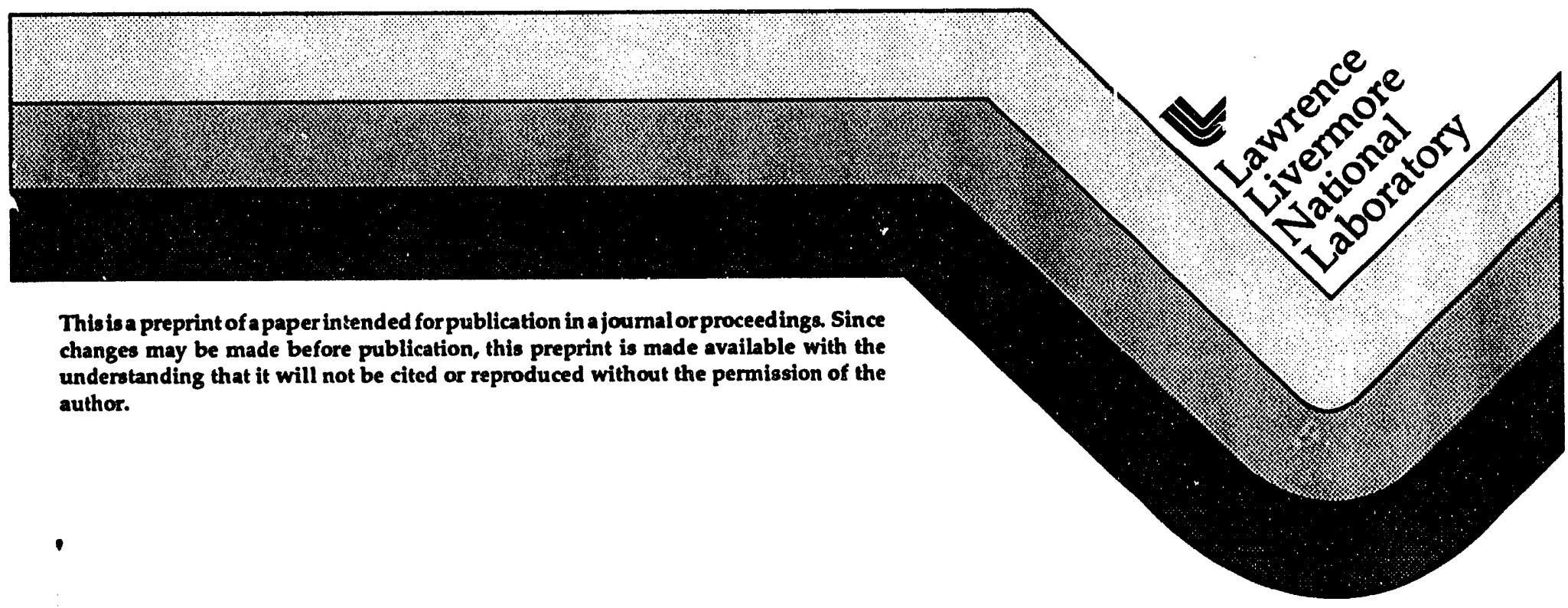

This is a preprint of a paper intended for publication in a journal or proceedings. Since changes may be made before publication, this preprint is made available with the understanding that it will not be cited or reproduced without the permission of the author. 


\section{DISCLAIMER}

This document was prepared as an account of work sponsored by an agency of the United States Government. Neither the United States Government nor the University of California nor any of their employees, makes any warranty, express or implied, or assumes any legal liability or responsibility for the accuracy, completeness, or unifulness of any information, apparatus, product, or process disclosed, or represents that its use would not infringe prifately owned rights. Reference herein to any spectific conumercial products, process, or service by trade name, trademark, manufacturer, or otherwise, does not necessarliy constitute or imply its endorsement, recommendation, or favoring by the United States Government or the University of Callfornia. The viewsand opinions of authors expressed herein do not necessarily state or reflect those of the United States Government or the University of California, and shall not be used for advertising or product endorsement purposes. 
MODELLING OF INTENSE LINE RADIATION FROM LASER-PRODUCED PLASMAS

Yim T. Lee and M. Gee

Lavrence Livermore National Laboratory

Livermore, CA, U.S.A.

\section{INTRODUCTION}

Resonance line radiation has been observed in many laser-produced plasma experiments. These lines which are emitted by a difole transition from an excited state to ground state are usually the brightest in the spectra. Measured intensity ratios have been used to estimate plasma temperature and density $1-11$. However, for these measurements to be useful, the plasma must be optically thin to the lines. Micro-dot targets ${ }^{4-5}$ have been used to produce plasmas with small enough optical depths in the transverse direction to allow the lines to escape.

Re-absorption (i.e. trapping) of resonance lines in a plasma will not just make the interpretation of an emission spectrum difficult, but it can affect the plasma condition. For an example, the plasma can become hotter and more ionized. However, for low - Z plasmas such as aluminum and chlorine which are of interest here, the effect of trapping of optically thick lines on the plasma temperature is usually small. This is because radiative loss in these plasmas is only a small fraction of the total energy. However, trapping will enhance populations of ions in excited states. This usually results in a more ionized plasma because ionization from excited states dominates over other collisional and radiative processes at the density of laser-produced plasmas.

Trapping of optically thick resonance lines can also have an important effect on the performance of a $x$-ray laser. It populates the lower state of a laser transition, thereby resulting in a laser with $a$ lower or no gain. The effect of trapping of optically thick lines on the gain coefficients of both recombination and collisional excitation pumped soft $x$-ray lasers has been investigated recently $12-13$. It was found that large ion velocity-gradients usually generated in laser-produced plasmas play an important roll in the transfers of optically thick lines. As a result of Doppler shifts, line absorption occurs only over the distance at which the shifts equal to the width of a line. The importance of such an effect on transfers of optically thick lines in astrophysical plasmas was pointed out by V. V. Sobolve in $1957^{14}$. In the laser-produced plasmas which have been used as amplifying media, the distance over which absorption occurs is also much less than the plasma size. This means absorption occurs only locally. As a result, escape probability should give good estimates of the line-transfers in these 
plasmas. This was pointed out several years ago $15-16$ and was recently shown to be true by comparing the results from escape probability calculations iv that from detailed line-transfer calculations in both planar ${ }^{12}$ and cylindrical 17 geometries.

In this paper, we discuss modelling of Lyman- $\alpha$ (i.e. Ly- $\alpha$ ) radiation emitted from laser-produced plasmas. We are interested in the application of one of these line radiations to pump a transition of an ion in a different plasma spatially separated from the emitting source. The interest is in perturbing the plasma rather than just probing it as in some backlighting experiments. As a result of pumping, the populations of certain excited levels are inverted. The resulting gain coefficients depend strongly on the population inversion density which in turn depends on the brightness of the pump radiation. As a result, we must produce an intense bright radiation source. In addition, to pump a transition effectively, we also need a pump line with a width larger than the mismatch of the resonance since the widths of the pumped transitions are rather narrow.

As an example on the modelling of resonance line radiation, we consider the Ly- $\alpha$ lines of aluminum emitted from laser-pfoduged plasmas. Recently, one of these lines has been proposed $18-19$ as a pump line for the $2 \mathrm{~s}-5 \mathrm{p}$ transitions in lithium-like iron. As a result of pumping, population inversions and hence gains are possible for the transitions between the $n-5$ and $n=4$ levels. Recently, we have performed gain calculations of $f i$ thium-like iron ions in a steady-state plasma or an exploding-foil target ${ }^{18}$. These results show that gain coefficients of $3-5 \mathrm{~cm}^{-1}$ are achievable in an exploding-foil plasma where the lithium-like ions are pumped by a line with brightness greater than $10^{12}$ $\mathrm{Wcm}^{-2}$.

To produce such an intense line radiation, we consider a source to be a massive slab target which is irradiated by high-power optical laser beams. In particular, we investigate the following two types of targets: $10 \mu \mathrm{m}$ aluminum slab and $1 \mu \mathrm{m}$ aluminum foil on top of $15 \mu \mathrm{m} \mathrm{CH}$ slab. These slab targets have been used in many laser-interaction experiments which measure shockwaves 20 -ray conversion 21 , and intense $\mathrm{K}$ - and L-shel1 line emissions 22 . In a $10 \mu \mathrm{m}$ slab target, the Ly- $\alpha$ lines are mostly emitted from the front side of the slab which is irradiated directly by the optical laser. In a $1 \mu \mathrm{m}$ aluminum foil target, the line radiation is mainly emitted from the back side of the foil which is between the aluminum and $\mathrm{CH}$. In these targets, we find that in order to produce an intense bright line the plasma condition has to be such that the scurce function of the lines would peak inside the critical density.

In next section, we discuss the lithium-like iron laser scheme in which the $5 p$ levels are pumped by aluminum Ly- $\alpha$ radiation emitted from a laser-produced plasma. The line brightness which is needed for pumping to achieve population inversions and gains are considered. Section III presents calculations of the Ly- $\alpha$ lines emitted from an aluminum slab target. We also show how these line brightness depends on the optical laser irradiance on a target.

\section{APPLICATION OF INTENSE BRIGHT RESONANCE LINE RADIATION}

In this section, we discuss application of resonance line radiation in the pumping of a x-ray laser. Here, as an example, we discuss pumping of the 5p levels in lithium-like iron ions by a Ly- $\alpha$ line ( $2 p_{3 / 2}-1 s_{1 / 2}$ transition) of aluminum. This resonance was first discovered by Lee, et al. in $1988^{18}$. Several other line pumped laser 
schemes in which lithium-like ions are photgexcited by either Ly- $\alpha$ or He- $\alpha$ radiation have been proposed recently ${ }^{19}$.

A simplified energy level diagram for lithium-like iron is given in Figure 1. Also shown in the figure are the wavelengths of the pumping and pumped transitions. These wavelengths match better than a few parts in ten thousands. In this laser scheme, pumping of the $5 \mathrm{p}$ levels will result in populating the other $n=5$ levels such as $5 d$, $5 f$, and $5 g$ because of strong collisional mixing among them. As a result of pumping, the populations of the $n=5$ and $n=4$ levels are inverted. The transitions between these levels in lithium-like iron have wavelengths of approximately $70 \AA$.

To achieve lasing on these transitions, explgding foil targets have been proposed as possible laser amplifying media 18 . These plasmas are usually produced by irradiating two opposite high-power optical laser beams in line focus op a thin foil with thickness of a $1000 \AA$. Previous calculations 18 have shown that the $5 \mathrm{~g}-4 \mathrm{f}$ transitions in lithium-like iron have gains of $2-3 \mathrm{~cm}^{-1}$ if an ion is pumped by a line with intensity greater than $10^{12} \mathrm{Wcm}^{-2}$. Among the dipole allowed 5-4 transitions, the $5 \mathrm{~g}-4 \mathrm{f}$ transitions have the highest gains because of their large oscillator strengths.

In these gain calculations, it is assumed that the pumped and pumping transitions are overlapped as shown in Figure 2. The widths of the pumped transitions are determined by thermal Doppler broadening because Stark and opacity broadenings are negligible at the density of the exploding foil targets. Assuming an ion temperature of $500 \mathrm{eV}$, we calculate the width of the $2 s_{1 / 2}-5 p_{1 / 2}$ transition in lithium-1ike iron to be $0.34 \mathrm{eV}$. This approximately equals the mismatch of the resonance shown in Figure 1 . Therefore, the width of the pump line has to be larger that. In next section, we show that it is possible for laser-produced plasmas to emit Ly- $\alpha$ lines with widths which are 4-5 times larger than their thermal Doppler widths.

\section{CALCULATION OF LY- $\alpha$ RADIATION FROM ALUMINUM SLAB TARGETS}

To produce intense bright Ly- $\alpha$ lines, we consider a possible source to be a slab target which is irradiated by a high-power optical laser beam. In our calculations, we have used both a $0.35 \mu \mathrm{m}$ wavelength laser beam in a gaussian pulse of $450 \mathrm{ps}$. FWHM and a 0.53 $\mu \mathrm{m}$ wavelength laser beam in a flat top pulse of $1 \mathrm{~ns}$. Two types of targets are used: a $10 \mu \mathrm{m}$ aluminum slab (target A) and a $15 \mu \mathrm{m} \mathrm{CH}$ $\mathrm{slab}$ (target B) with $1 \mu \mathrm{m}$ of aluminum foil on top. In target A, line radiation is mostly emitted from the front surface of a slab which is heated directly by an optical laser beam. In target $B$, line radiation is mostly emitted from the back surface of a $\mathrm{CH}$ slab. The intensity of the line radiation at the front side of the aluminum foil is much smaller and the line profile is significantly Doppler shifted.

Our calculations of Ly- $\alpha$ line radiation emitted from these targets consist of two steps. First, we model absorption of an optical laser beam and heating of a target by using the hydrodynamics code LASNEX ${ }^{23}$. In these one-dimensional calculations, the plasmas are assumed to expand into a spherical ccne to account for their diverging flows. Calculations done this way have been found to give reasonable results when they are compared to two-dimensional simulations ${ }^{24}$. In the second step of the calculations, hydrodynamics output such as temperature, density, and continuum radiation are input together with an atomic model to the kinetics code XRASER ${ }^{25}$ to compute detailed transfers of optically thick resonance lines. The line-transfers are calculated by using a 
multi-angles and multi-frequencies numerical method ${ }^{12}$. We have also assumed complete re-distribution of the line profile in the calculations. In these calculations, the intrinsic line width is assumed to be the thermal Doppler width. Other contributions such as Stark broadening and electron broadening are in general very small at the density of the slab target. However, as given later, the widths of the emission lines from these targets are much wider than the intrinsic widths of the lines because of broadening by opacity and ion velocity gradients in a plasma.

As an example of a typical LASNEX calculation, we plot in Figure 3 the temperatures at different zones of a $10 \mu \mathrm{m}$ aluminum slab target at a time near the peak laser irradiance. The target is assumed to be irradiated by a $0.35 \mu \mathrm{m}$ wavelength laser beam in a gaussian pulse of $450 \mathrm{ps}$. FWHM and at a peak intensity of $8 \times 10^{14} \mathrm{Wcm}^{-2}$. The charge-state distribution at different zones of the target is shown in Figure 4. We see that hydrogen-like population is less than 108 throughout most of the underdense plasma and reaches a maximum value of 408 inside the overdense plasma. However, near the front surface of the slab, hydrogen-like population starts to increase with the decrease in electron density. This is because the contribution of ionization from excited states decreases very fast with density, approximately as a square of density. The recombination rates, on the other hand, decrease only linearly with density. Therefore, the plasma should recombine with the decrease in density as shown in Figure 4.

To estimate the importance of trapping of resonance lines in a slab target, we have calculated the optical depths at different locations in the target. The optical depth just inside the critical density are about 20 at a time near the peak irradiance of a laser pulse. This shows that most of the lines emitted inside the critical density are trapped. In addition, as shown later, the transfers of these lines are very important in determining the line emission from the target.

In Figures 5a-5b, we plot the source function of the Ly- $\alpha$ lines inside an aluminum slab target at a time near the peak laser irradiance. In these figures, we express brightness in an unit of photons/mode, $N(h \nu)$. Radiation intensity in an unit of $\mathrm{Wcm}^{-2}$ can be obtained from $\mathrm{N}(\mathrm{h} \nu)$ as,

$$
\begin{aligned}
\mathrm{I}_{\mathrm{h} \nu} & =\left[8 \pi(\mathrm{h} \nu)^{3} / \mathrm{c}^{2}\right] \mathrm{N}(\mathrm{h} \nu) \\
& \simeq 6.332 \times 10^{4}(\mathrm{~h} \nu)^{3} \mathrm{~N}(\mathrm{~h} \nu) \mathrm{W} /\left(\mathrm{cm}^{2}-\mathrm{eV}\right)
\end{aligned}
$$

where $h \nu$ is the photon energy in electron-volt. As an example, a resonance line with an energy of $1729 \mathrm{eV}$, a brightness of 0.005 photons/mode and a line width of $1 \mathrm{eV}$ is equivalent tc an intensity of approximately $10^{12} \mathrm{Wcm}^{-2}$. Figure 5 a shows the results for a $10 \mu \mathrm{m}$ aluminum slab which is irradiated by a laser pulse with an intensity of $8 \times 10^{14} \mathrm{Wcm}^{-2}$ and Figure $5 \mathrm{~b}$ shows the results corresponding to the laser intensity of $1.6 \times 10^{14} \mathrm{Wcm}^{-2}$. Comparing these results, we find that irradiating the target with a higher laser irradiance would produce a plasma such that the source function of the lines would have larger values. In addition, these values are located at a higher density part of the overdense plasma.

In the slab targets, the source function of the Ly- $\alpha$ lines depends on the populations of the $2 p$ levels of hydrogen-1ike aluminum ions. Since these levels are mainly populated by electron collisional excitation from the ground state, their populations are approximately proportional to the collisional excitation rates and to the ground state population of hydrogen-like aluminum. The collisional rates depend on both the plasma temperature and density. Because of the linear dependence 
of the collisional rates on the electron density, the plasma should be as dense as possible. The dependence of the collisional rates on the temperature is more complicated. For dipole allowed transitions such as the $1 s_{1 / 2}-2 p_{1 / 2}$ and $1 s_{1 / 2}-2 p_{3 / 2}$, the rate coefficients usually are the largest near the temperatures which equal to the excitation energy. At a temperature below one half of the excitation energy, the rate coefficients decrease exponentially with temperature. Therefore, the plasma should have a temperature between one half of the excitation energy and the excitation energy.

The dependence of the ground state populations of hydrogen-like aluminum ions on the condition of a plasma is also complicated. This is because the plasma is at non-Local Thermal Equilibrium. In addition, trapping of optically thick resonance lines can deplete the ground state populations. According to our calculations, an aluminum plasma with a temperature in a range of $800-1200 \mathrm{eV}$ and at a density of a few times $10^{22} \mathrm{~cm}^{-3}$ would have hydrogen-like populations at least of 108 . At a temperature above $1200 \mathrm{eV}$, the plasma will be over ionized, containing mostly fully stripped ions. For an example, hydrogen-like aluminum populations in a plasma with a temperature greater than $1500 \mathrm{eV}$ and an electron density of $10^{22} \mathrm{~cm}^{-3}$ are less than a few 8 .

The density consideration of a plasma suggests that, as a source of bright Ly- $\alpha$ lines, massive slab targets are better than thin foil targets. These foil targets which are burned through by the optical laser beams produce rather uniform but low density plasmas. Typical densities in a foil target which is irradiated by a laser driver with $0.35 \mu \mathrm{m}$ wavelength are a few times $10^{20}-10^{21} \mathrm{~cm}^{-3}$. These densities are more suitable as a laser amplifying medium than as a source of line radiation. In addition, thin foil targets also emit line radiation which is significantly Doppler shifted. Such resonance lines have been observed in both experiments and computer simulations ${ }^{26}$.

Figure 6 shows the source functions of the Ly- $\alpha$ lines in a $1 \mu \mathrm{m}$ aluminum foil of target $B$. In the calculations, the target was assumed to be irradiated by a $0.53 \mu \mathrm{m}$ wavelength laser beam in a $1 \mathrm{~ns}$ flat top pulse with an intensity of $4.0 \times 10^{14} \mathrm{Wcm}^{-2}$. To model the radiation at the boundary between the aluminum and the $\mathrm{CH}$, we had to fine zone the aluminum at the boundary. Typically, the first few zones at the surface were choosen so they will have optical depth less than 0.1 at a time near the peak irradiance of a laser pulse.

Also shown in Figures $5 a-5 b$ and 6 are the maximum intensity of the Ly- $\alpha$ line radiation at each zone of the slab target. The intensity is averaged over solid angles. In target $A$, the maximum intensity at the front surface of the slab is 6-7 times larger than the local source function. This is because the intensity is characteristic of the values of the source function which is within several optical depths from the surface of the slab target. Near the surface, most of the line radiation are transfered here from the dense part of the target where the lines are first emitted. In target $B$, the maximum intensity at the front surface of the foil is approximately 10 times larger than the local source function. However, at the back surface of the foil, the maximum intensity is smaller than the local source function by as much as 708 . We can understand this result as follows. Since there is no radiation entering the aluminum foil at the boundary between the aluminum and the $\mathrm{CH}$, the angle averaged intensity at the first zone of the boundary is at least a factor of two less than the intensity at an angle perpendicular to the target surface. If the line radiation is emitted isotropically, the angle averaged intensity would be exactly half of the intensity at any angle. Taking this into consideration, we see that the intensity at an angle 
perpendicular to the target surface is actually larger than the local source function. Again, as in the front surface of the foil, part of the intensity is characteristic of the values of the source function inside the target surface.

Figures $7 a-7 b$ present the angle averaged intensity profile of the Ly- $\alpha$ lines at two zones 40 and 118 of a $10 \mu \mathrm{m}$ aluminum slab target. The laser irradiance on the target is the same as in Figure 5a. Zone 40 is inside the critical density, while zone 118 is the first zone at the surface of the slab. In our calculations, the first zone has been choosen so it will have a small enough optical depth to ensure the line emission intensity from the surface of the slab would be the same as the line intensity in the first zone. Therefore, Figure $7 \mathrm{~b}$ also represents the line emission profile from the target.

The Ly- $\alpha$ lines consist of two fine-structure components, namely the $2 \mathrm{p}_{1 / 2^{-1}} \mathrm{~s}_{1 / 2}$ and $2 \mathrm{p}_{3 / 2^{-1}} \mathrm{~s}_{1 / 2}$ transitions. These two transitions are separated in energy of approximately $1.3 \mathrm{eV}$. In Figures $7 a-7 b$, zero photon energy corresponds to the energy of the $2 p_{1 / 2}-1 s_{1 / 2}$ transition, while the arrow points at the energy of the $2 \mathrm{p}_{3 / 2}-\mathrm{s}_{1 / 2}$ transition.

Inside the critical density, the Ly- $\alpha$ lines are red shifted by an amount of approximately $0.2 \mathrm{eV}$. This is due to the contribution of these lines which are first emitted inside the ablation front. These lines are red shifted because the ions are moving away from the front surface of the slab. According to LASNEX calculations, ions inside the ablation surface have velocity which can Doppler shift the line to a lower energy by 0.2 eV. The intensity profile at the surface of the slab is also red shifted, but by an amount of $0.3-0.4 \mathrm{eV}$.

Figures $7 a-7 b$ show that the width of the Ly- $\alpha$ lines is approximately $3.7 \mathrm{eV}$ inside the critical density and approximately $3.5 \mathrm{eV}$ at the surface of the slab. This suggests that the width of each fine-structure component is at least of $2.0 \mathrm{eV}$. Since the largest ion temperature inside the critical density is approximately $1200 \mathrm{eV}$ according to LASNEX calculations, this means that the thermal Doppler width should be no more than $0.80 \mathrm{eV}$. Therefore, the results in these figures suggest that the lines are broadened by opacity and ion velocity gradients.

At the surface of the slab, the values of the intensity profile near the line-center of the $2 \mathrm{p}_{1 / 2}-1 \mathrm{~s}_{1 / 2}$ transition are larger than that near the line-center of the $2 \mathrm{p}_{3} / 2-1 \mathrm{~s}_{1 / 2}$ transition. Since the $2 \mathrm{p}_{3 / 2}$ level population is a factor of two greater than the $2 \mathrm{p}_{1 / 2}$ level population and the radiative rates for the two transitions are the same, one might expect that the line emission intensity of the $2 \mathrm{p}_{3} / 2^{-1 s_{1}} / 2$ transition would be higher than the intensity of the $2 \mathrm{p}_{1 / 2}-1 \mathrm{~s}_{1 / 2}$ transition. However, as discussed previously, most of the line emission come from the high density part of the plasma. Therefore, line absorption plays a very important roll in determining the line intensity profile at the surface. The absorption cross section of the $2 \mathrm{p}_{3 / 2}-1 \mathrm{~s}_{1 / 2}$ transition is a factor of two larger than that of the $2 \mathrm{p}_{1 / 2^{-1}} \mathrm{~s}_{1 / 2}$ transition. As a result, there will be more absorption of the high energy component of the Ly- $\alpha$ lines. This explains why the maximum value of the line emission profile is near the low energy component of the Ly- $\alpha$ lines.

Figure $7 \mathrm{~b}$ shows that at the high energy side of the profile there is a small peak at approximately $2 \mathrm{eV}$ above the line-center of the $2 \mathrm{p}_{3 / 2}-1 \mathrm{~s}_{1 / 2}$ transition. In our calculations, we have found that the line emission profile would have that structure if the laser irradiance on 
the target is greater than $4 \times 10^{14} \mathrm{Wcm}^{-2}$. As the laser irradiance on the target increases, the location of the peak also shifts toward higher energy. Based on these results, we see that the peak represents the contribution from the source of the Ly- $\alpha$ lines which are emitted outside the critical density. At the laser irradiance of $4 \times 10^{14}$ $\mathrm{Wcm}^{-2}$, the critical density surface would be expanding at the velocity of a few times $10^{7} \mathrm{~cm} / \mathrm{sec}$. Ions moving at such a velocity would emit lines which are blue shifted by about $2 \mathrm{eV}$. This agrees with the results in Figure $7 b$.

In Figure 8, we show how the brightness of the Ly- $\alpha$ emission from a $10 \mu \mathrm{m}$ aluminum target would depend on the optical laser irradiance. To show the emission intensity profile, we plot in Figure 8 the maximum intensity and the line-center intensity at the first zone of the surface of the slab. Here, by line-center intensity, we mean the line brightness at the photon energy of the $2 \mathrm{p}_{3 / 2}-1 \mathrm{~s}_{1 / 2}$ transition. In these calculations, we assume a $0.35 \mu \mathrm{m}$ wavelength laser driver in a gaussian pulse of $450 \mathrm{ps}$. FWHM. Also shown in the figure is the maximum intensity of the Ly- $\alpha$ lines in the slab target. This intensity is several times brighter than the maximum intensity at the surface of the slab. This is because most of the radiation emitted inside the slab are trapped, but only a small fraction of it can escape from the target. In addition, the maximum intensity of the Ly- $\alpha$ lines inside the target increases very fast with the irradiance of the optical laser driver.

As a function of the optical laser irradiance on a target, the intensity profile at the surface increases much slower than the maximum intersity inside the target does. At the irradiance above $1.2 \times 10^{15}$ $\mathrm{Wcm}^{-2}$, the line-center intensity also starts to decrease. At these high irradiances, the plasma inside the ablation surface becomes hot enough to emit Ly- $\alpha$ line radiation. These lines, however, are red shifted because of the large ion velocity moving in the direction away from the surface. For an example, at a laser irradiance of $1.2 \times 10^{15} \mathrm{Wcm}^{-2}$, the temperature and ion velocity inside the ablation front could reach 900 $\mathrm{eV}$ and $2.0 \times 10^{7} \mathrm{~cm} / \mathrm{sec}$, respectively. Ions moving with such velocity will emit Ly- $\alpha$ lines which are red shifted by as much as $2.5 \mathrm{eV}$. Such a shift is about the same as the line width of the $2 \mathrm{p}_{3 / 2}-1 \mathrm{~s}_{1 / 2}$ transition. This explains why the line-center intensity decreases with laser irradiance above $1.2 \times 10^{15} \mathrm{Wcm}^{-2}$ as shown in Figure 8 .

The results in Figure 8 suggest that pump lines with brightness of about .003 photons/mode are achievable using laser-produced plasmas. To produce a much brighter pump line would require more sophisticated target designs. That is beyond the scope of the present paper. However, a pump line with brightness of .003 photons/mode, according to previous calculations, can be used to pump the $5 p$ levels in lithium-like iron to achieve $5:$ is of $2-3 \mathrm{~cm}^{-1}$ in the $5 \mathrm{~g}-4 \mathrm{f}$ transitions. These values for the gains are certainly measurable in an experiment.

\section{Acknowledgments}

I am grateful to the following colleagues for many helpful discussions on several aspects of the work presented here: E. Alley, M. Howard, and H. Scott.

This work was performed under the auspices of the U. S. Department of Energy by the Lawrence Livermore National Laboratory under Contract W- 7405 - ENG -48 . 
1 B. K. F. Young et al., Phys. Rev. Lett. 61,2851 (1988).

2 J. P. Apruzese, D. Duston, and J. Davis, J. Q. S. R. T. 36, 339 (1986).

3 C. DeMichelis and M. Mattioli, Nucl. Fusion 21, 677 (1981).

4 M. J. Herbst et al., Rev. Sci. Instrum. 53, 1418 (1982).

5 P. G. Burkhalter et al., Phys. Fluids 26, 3850 (1983).

6 V. A. Boiko, I. Yu. Sobelev and A. Ya. Faenov, Fiz. Plazmy 10, 143 (1984) [Sov. J. Plasma Phys. 10, 82 (1984)]. 7 J. C. Gauthier et al., J. Phys. D 16,1929 (1983).

8 P. Alaterre et al., Opt. Commun. 49, 140 (1984).

9 M. H. Key et al., Phys. Rev. Lett. 44, 1669 (1980).

10 R. L. Kauffman, R. W. Lee, and K. Estabrook, Phys. Rev. A 35, 4286 (1987).

11 R. W. Lee, J. Q. S. R. T. 27, 87 (1982).

12 Y. T. Lee, et al., "Application of Escape Probability to LineTransfer in Laser-Produced Plasmas", Lawrence Livermore National Laboratory Report, UCRL-102219 (1989).

13 D. C. Eder, Phys. Fluids: B 1 (12), 2462 (1989).

14 V. V. Sobolev, Soviet Astr.-AJ 1, 131 (1987).

15 R. A. London, "1986 Laser Program Annual Report," Lawrence Livermore National Laboratory, CA, UCRL-50021-86 (1987).

16 G. Pert, J. Phys. B: Atom. Molec. Phys. 9, 3301 (1976).

17 D. C. Eder, private communication, Lawrence Livermore National Laboratory, CA.

18 Y. T. Lee, et al., J. Q. S. R. T. (1990, in press).

19 Y. T. Lee, et al., "Resonant Photopumping of Lithium-like Ions in Laser-Produced Plasmas", NATO-ASI volume on Non-Equilibrium Processes in Partially Ionized Gases (1990, in press).

20 For example, A. Ng, et al., Phys. Rev. Lett. 24, 2604 (1985).

21 For example, P. Alaterre, et al., Phys. Rev. A 34,4184 (1986).

22 For example, D. L. Matthews, et al., J. Appl. Phys. 54, 4260 (1983).

23 G. B. Zimmerman and W. L. Kruer Comments Plasma Phys. Controlled Fusion 11, 51 (1975).

24 W. C. Mead, et al., Phys. Rev. Lett. 47, 1289 (1981).

25 P. L. Hagelstein, Ph. D. thesis, Massachusetts Institute of Technology, (1981).

26 M. S. Maxon, et a1., "1985 Laser Program Annual Report," Lawrence Livermore National Laboratory, CA, UCRL-50021-85 (1986). 


\section{FIGURE CAPTIONS}

Fig. 1: A laser scheme based on pumping of lithium-like iron ions by using a Ly- $\alpha$ line of aluminum.

Fig. 2: Overlapping between the pump line radiation and the pumped transition.

Fig. 3: Electron temperature inside a $10 \mu \mathrm{m}$ aluminum slab at a time near the peak intensity of a laser beam. The laser beam which has a wavelength of $0.35 \mu \mathrm{m}$ is in a gaussian pulse of 450 ps FWHM and at an intensity of $8 \times 10^{14}$ $\mathrm{Wcm}^{-2}$. The arrow points at the zone where the critical density is located.

Fig. 4: Fractional populations of aluminum ions inside a $10 \mu \mathrm{m}$ slab at a time near the peak intensity of a laser beam. The laser parameters are the same as in Figure 1 . The arrow points at the zone where the critical density is located.

Fig. 5a: Maximum intensity and source function of aluminum Ly- $\alpha$ lines at a time near the peak intensity of a laser beam.

Fig. 5b: Maximum intensity and source function of aluminum Ly- $\alpha$ lines at a time near the peak intensity of a laser beam.

Fig. 6: Maximum intensity and source function of aluminum Ly- $\alpha$ lines at a time near the peak intensity of a laser beam.

Fig. 7a: Intensity profile of aluminum Ly- $\alpha$ lines at a zone number 40 which is inside the critical density surface of the slab. The arrow locates the line-center of the $2 \mathrm{p}_{3 / 2}-1 \mathrm{~s}_{1 / 2}$ transition.

Fig. 7b: Intensity profile of aluminum Ly- $\alpha$ lines at the surface of the slab target. The arrow locates the line-center of the $2 p_{3 / 2}-1 s_{1 / 2}$ transition.

Fig. 8: Maximum intensity and emission intensity of the aluminum Ly- $\alpha$ lines vs. optical laser driver irradiance. 


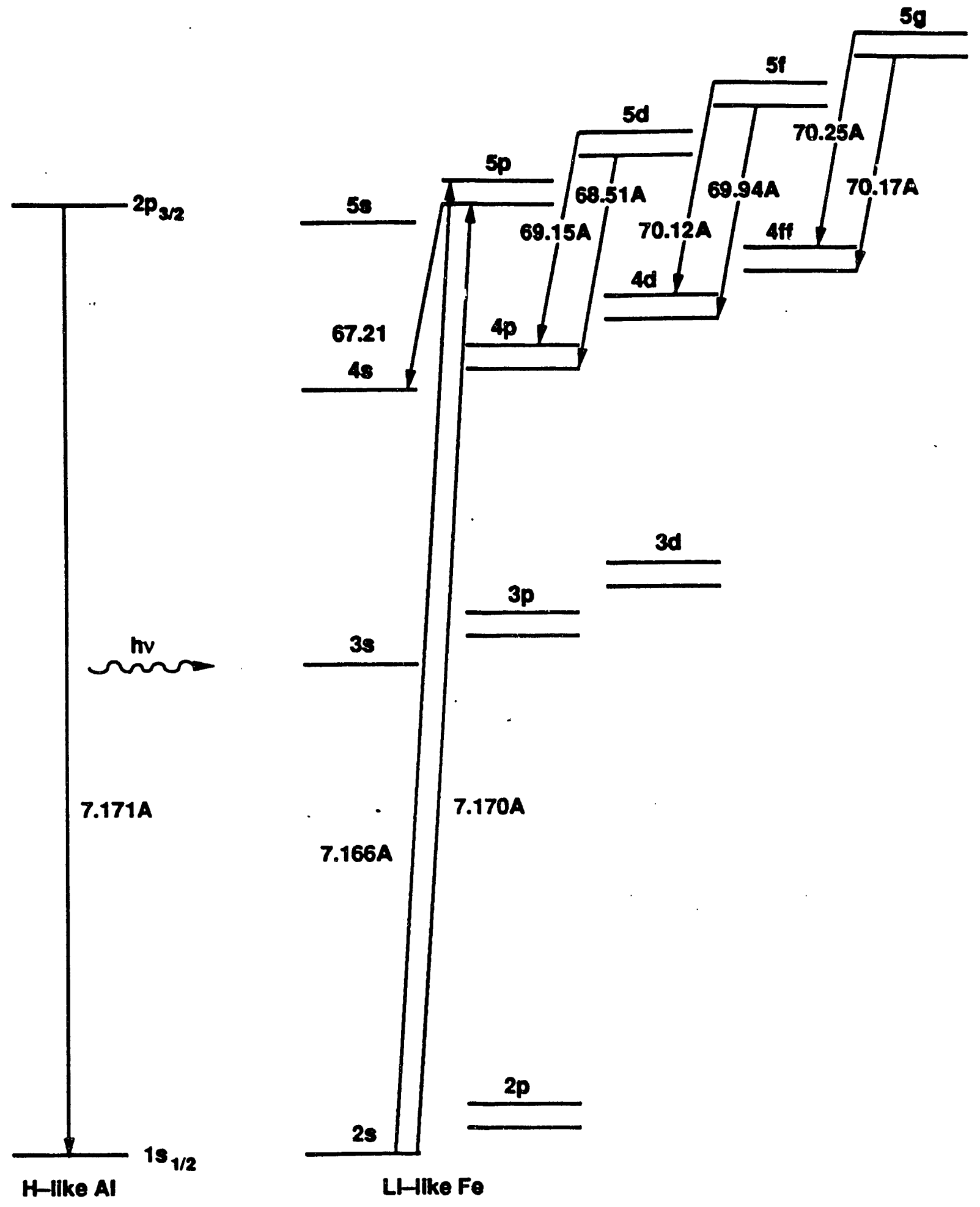

Fig. 1: A laser scheme based on pumping of lithium-like iron ions by using a Ly- $\alpha$ line of aluminum. 


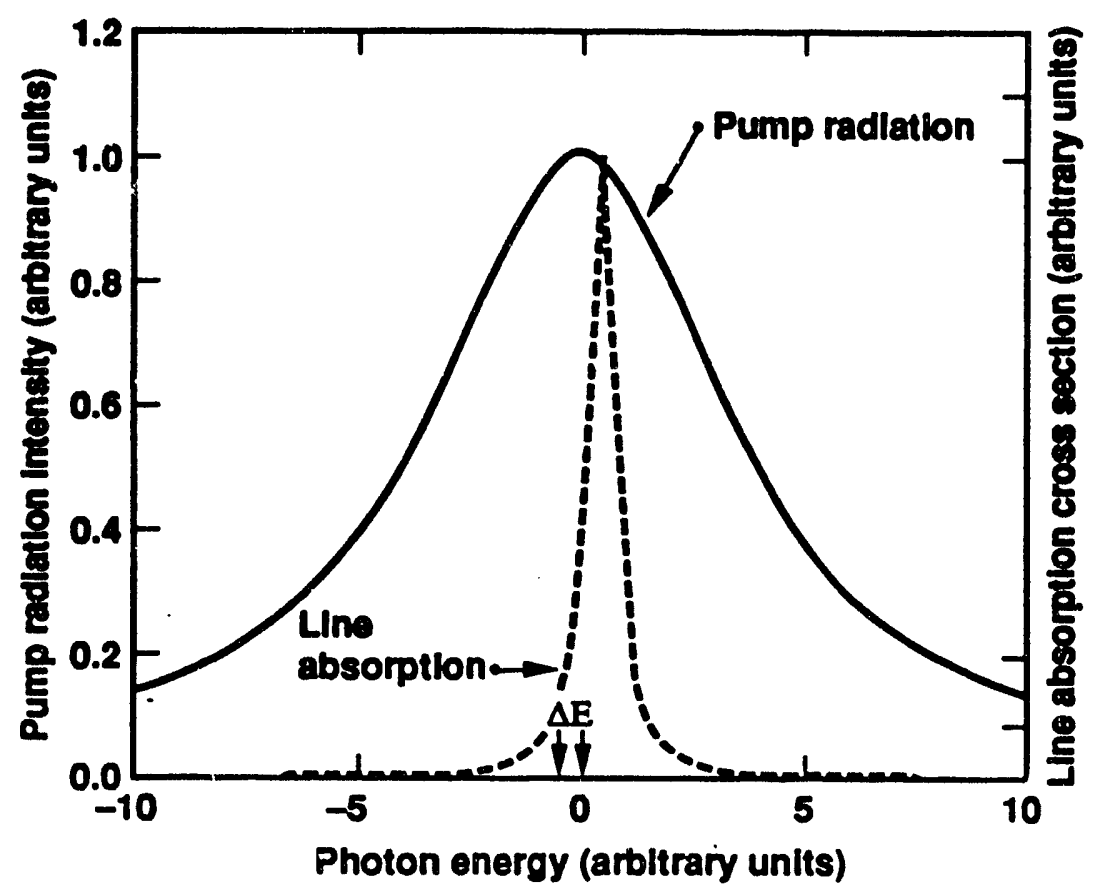

Fig. 2: Overlapping between the pump line radiation and the pumped transition. 


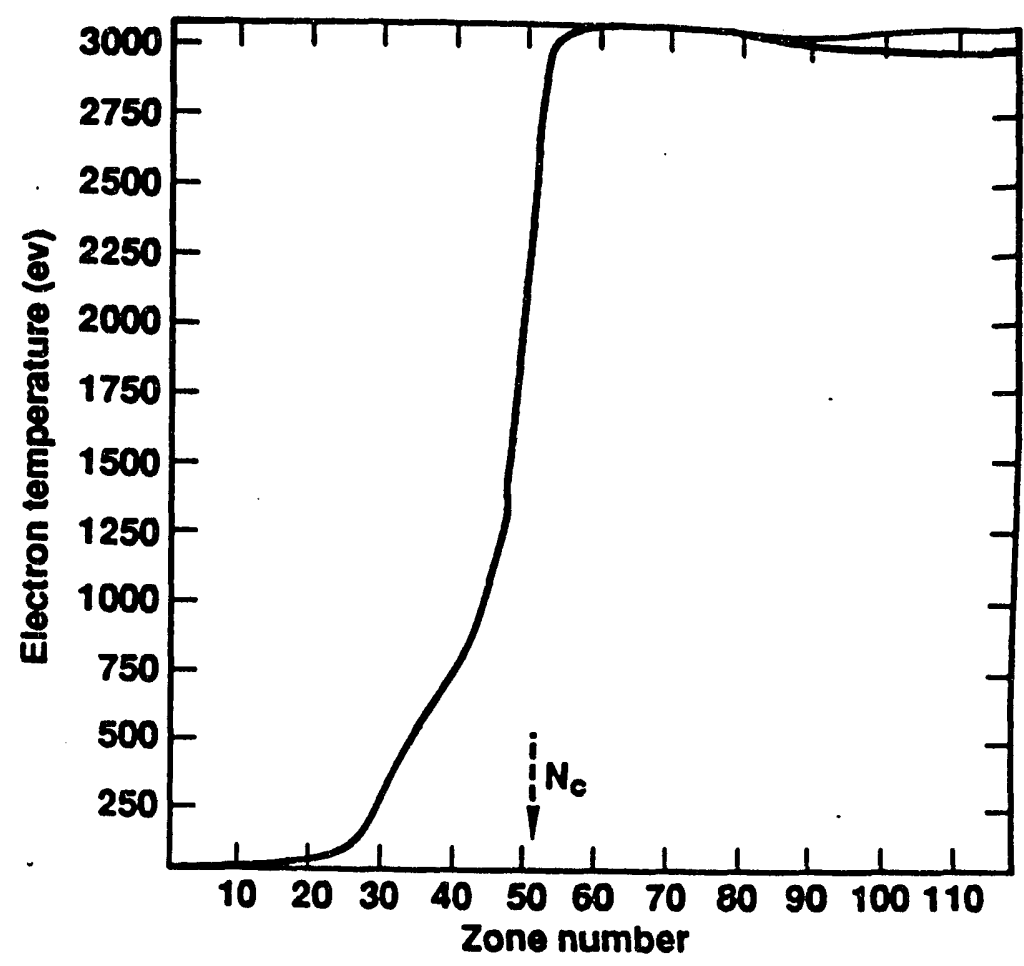

Fig. 3: Electron temperature inside a $10 \mu \mathrm{m}$ aluminum slab at a time near the peak intensity of a laser beam. The laser beam which has a wavelength of $0.35 \mu \mathrm{m}$ is in a gaussian pulse of 450 ps FWHM and at an intensity of $8 \times 10^{14}$ $\mathrm{Wcm}^{-2}$. The arrow points at the zone where the critical density is located.

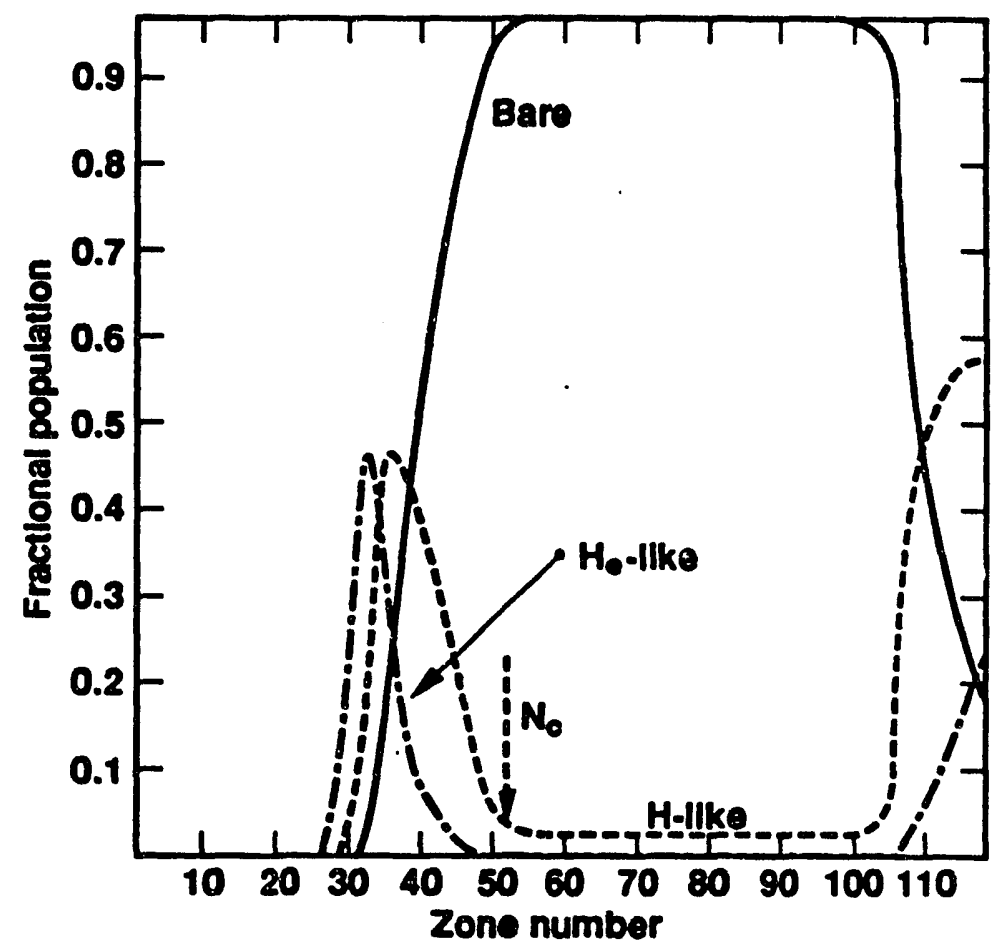

Fig. 4: Fractional populations of aluminum ions inside a $10 \mu \mathrm{m}$ slab at a time near the peak intensity of a laser beam. The laser parameters are the same as in Figure 1 . The arrow points at the zone where the critical density is located. 


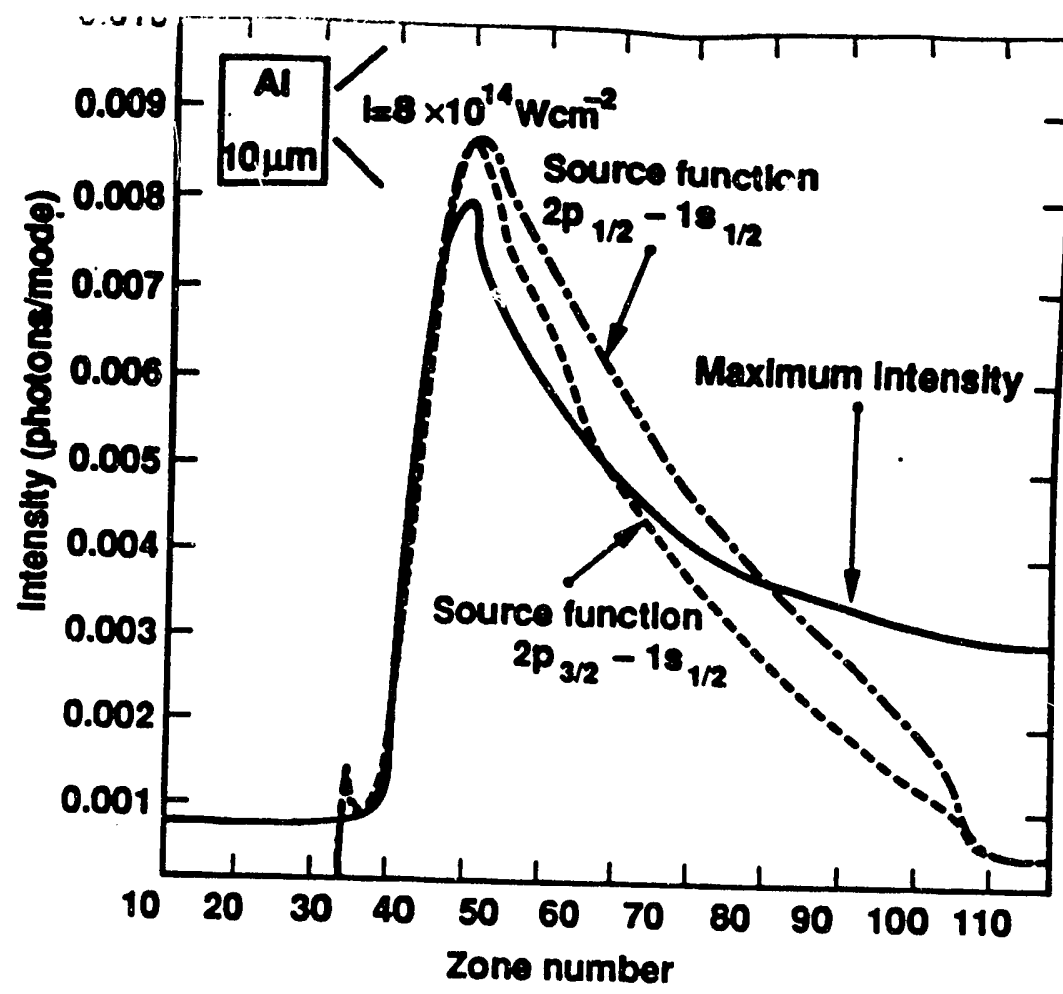

Fig. 5a: Maximum intensity and source function of aluminum Ly-a lines at a time near the peak intensity of
a laser beam.

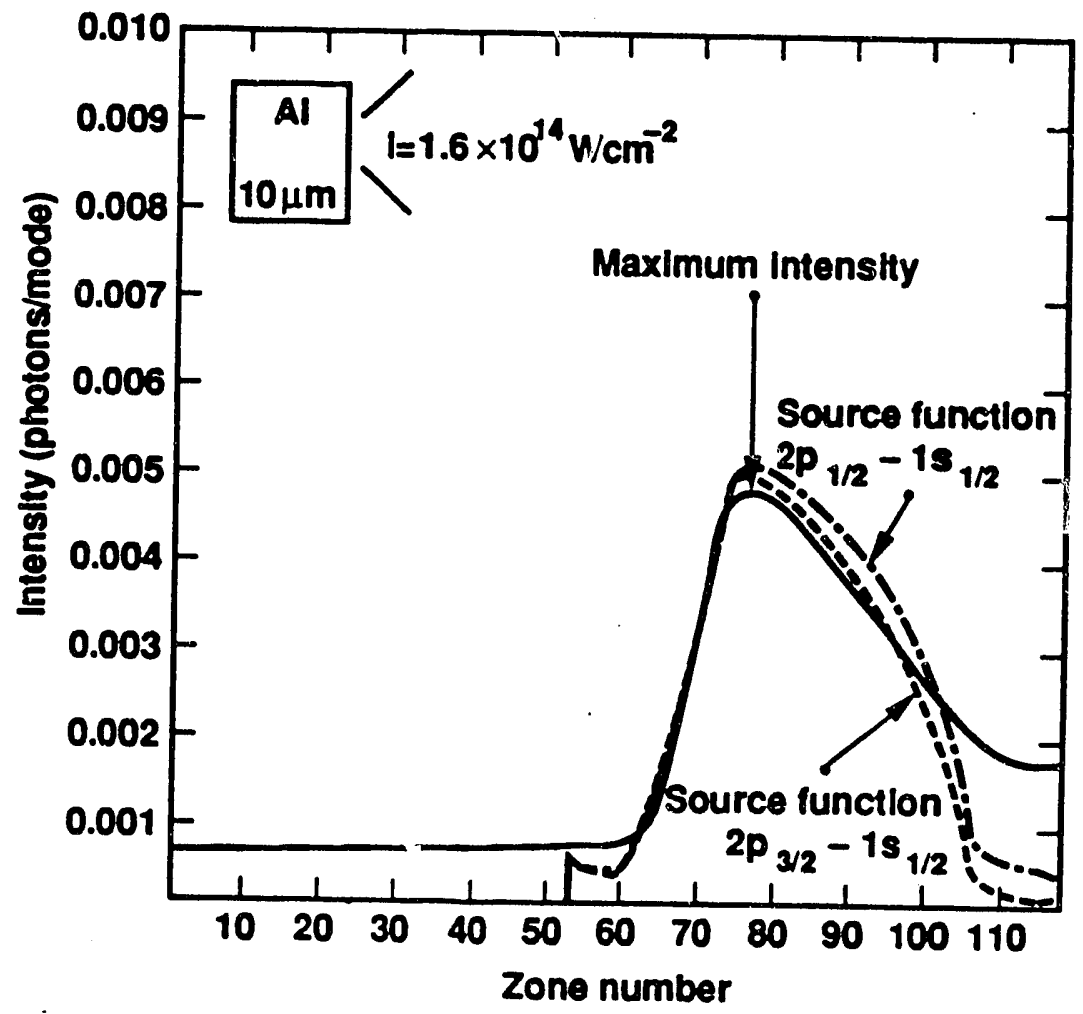

Fig. 5b: Maximum intensity and source function of aluminum Ly-a lines at a time near the peak intensity of a laser beam. 


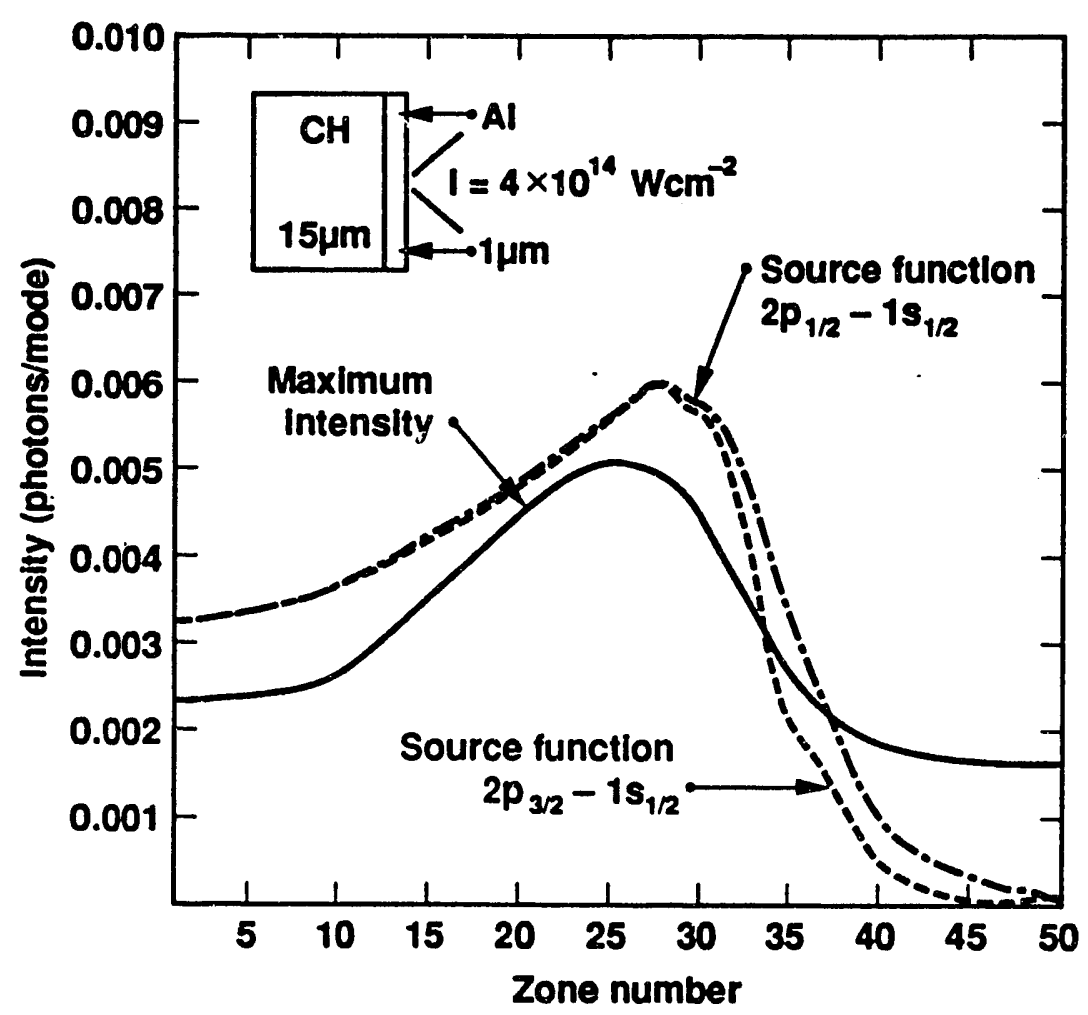

Fig. 6: Maximum intensity and source function of aluminum Ly- $\alpha$ lines at a time near the peak intensity of a laser beam. 


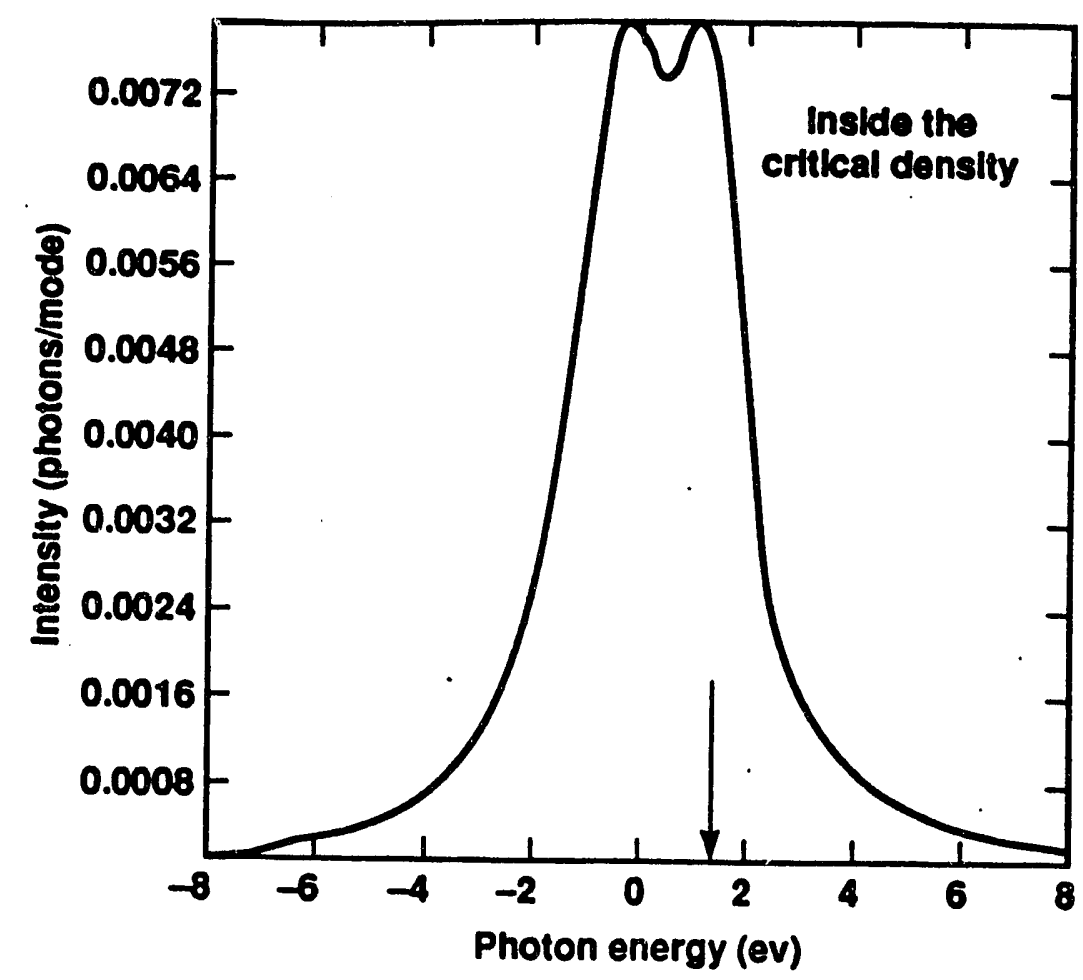

Fig. 7a: Intensity profile of aluminum Ly- $\alpha$ lines at a zone number 40 which is inside the critical density surface of the slab. The arrow locates the line-center of the $2 \mathrm{p}_{3 / 2}-1 s_{1 / 2}$ transition.

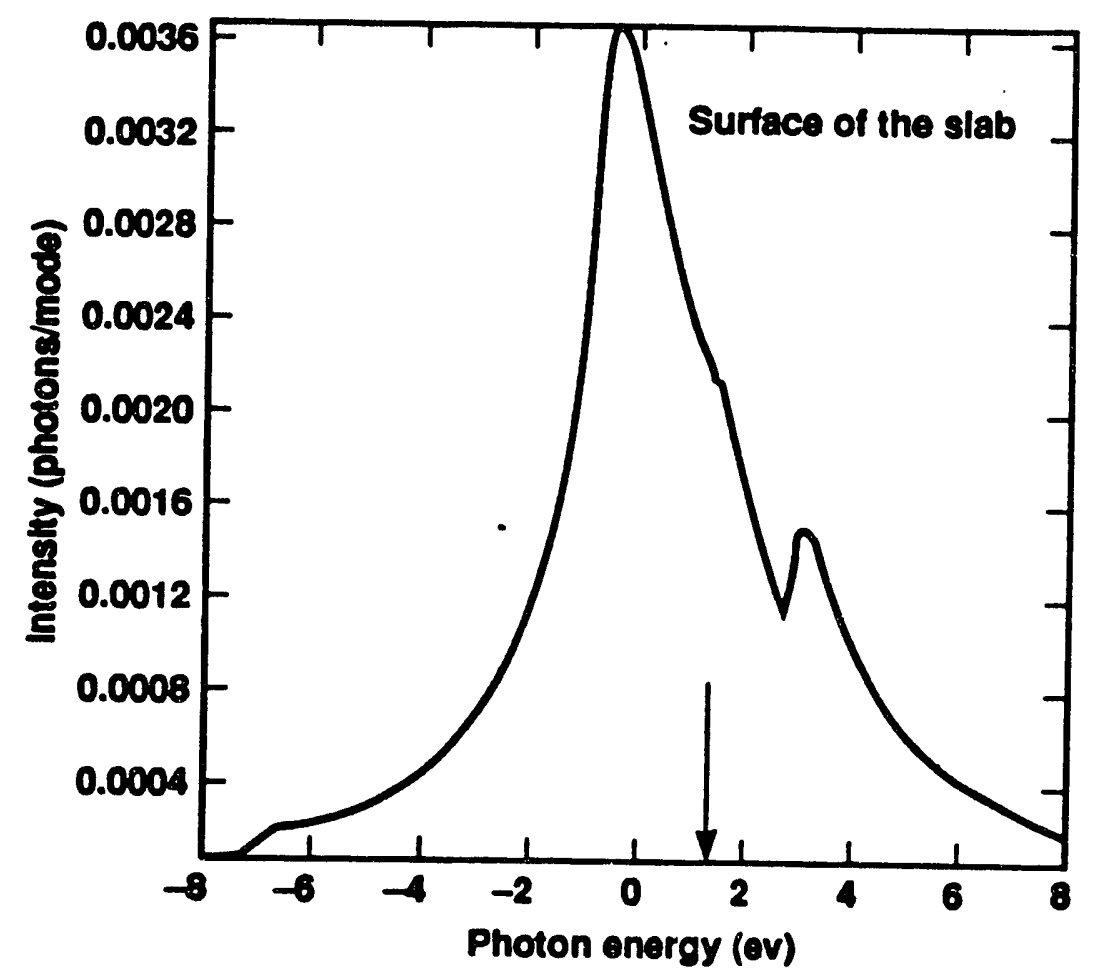

F1g. 7b: Intensity profile of aluminum Ly-a lines at 


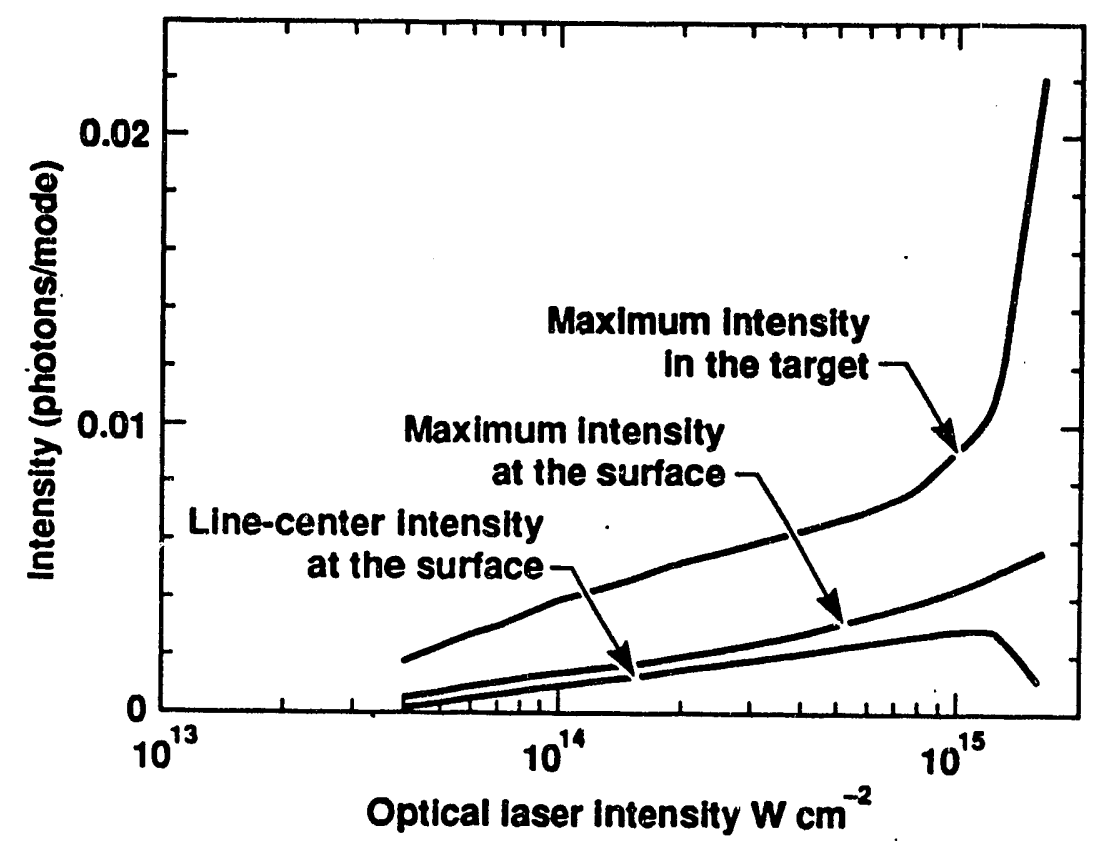

Fig. 8: Maximum intensity and emission intensity of the aluminum Ly-a lines vs. optical laser driver irradiance. 

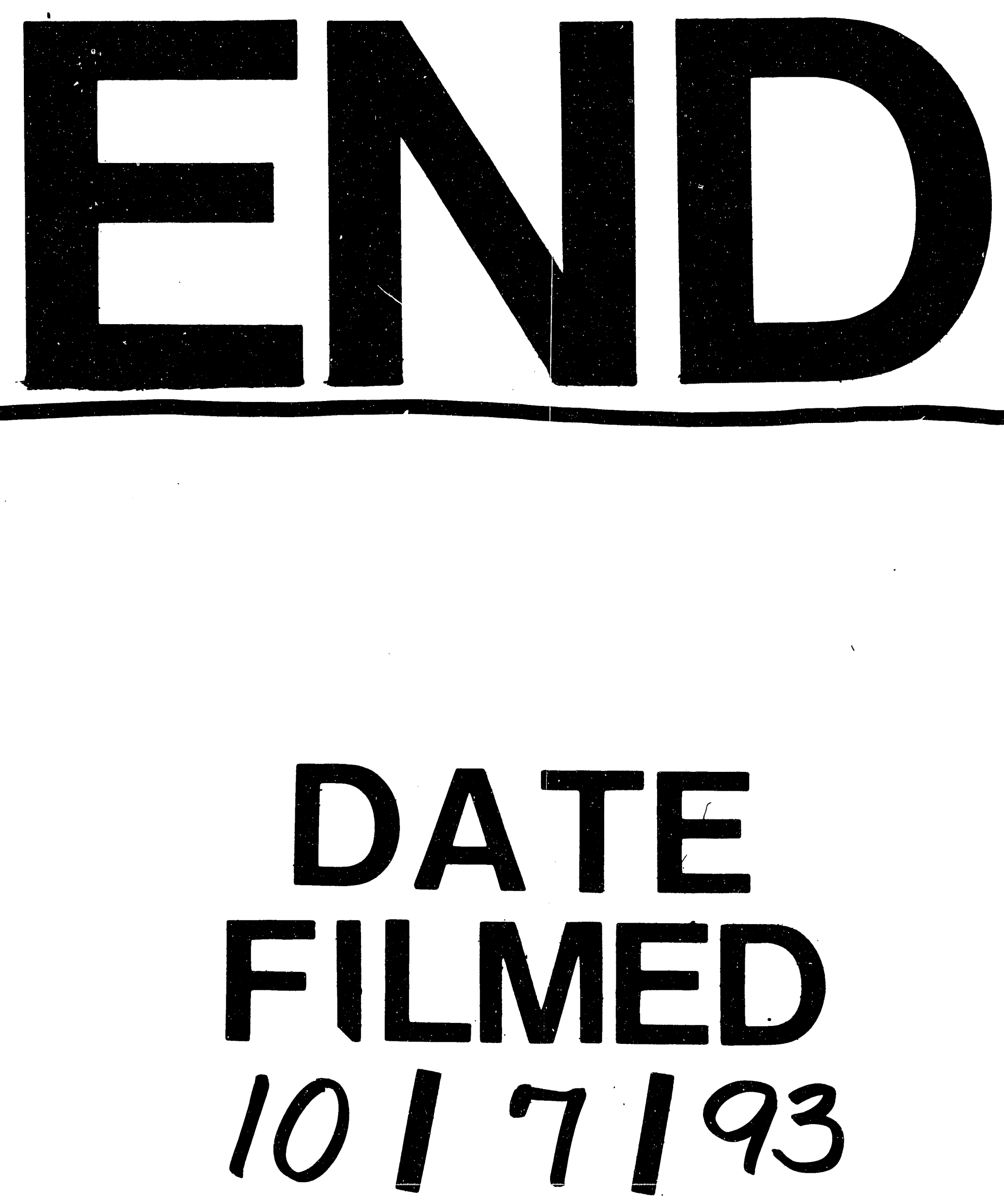
